\title{
Giant nonlocality in nearly compensated two-dimensional semimetals
}

\author{
S. Danz ${ }^{1}$ M. Titov, ${ }^{2,3}$ and B. N. Narozhny $\oplus^{1,4}$ \\ ${ }^{1}$ Institut für Theorie der Kondensierten Materie, Karlsruhe Institute of Technology, 76128 Karlsruhe, Germany \\ ${ }^{2}$ Radboud University Nijmegen, Institute for Molecules and Materials, NL-6525 AJ Nijmegen, The Netherlands \\ ${ }^{3}$ ITMO University, 197101 St. Petersburg, Russia \\ ${ }^{4}$ National Research Nuclear University MEPhI (Moscow Engineering Physics Institute), 115409 Moscow, Russia
}

(Received 11 February 2020; accepted 30 July 2020; published 18 August 2020)

\begin{abstract}
In compensated two-component systems in confined, two-dimensional geometries, nonlocal response may appear due to an external magnetic field. Within a phenomenological two-fluid framework, we demonstrate the evolution of charge flow profiles and the emergence of a giant nonlocal pattern dominating charge transport in a magnetic field. Applying our approach to the specific case of intrinsic graphene, we suggest a simple physical explanation for the experimental observation of giant nonlocality. Our results provide an intuitive way to predict the outcome of future experiments exploring the rich physics of many-body electron systems in confined geometries as well as to design possible applications.
\end{abstract}

DOI: 10.1103/PhysRevB.102.081114

The trend towards miniaturization of electronic devices requires a deeper understanding of the electron flow in confined geometries. In contrast to the electric current in household wiring, charge flow in small chips with multiple leads may exhibit complex spatial distribution patterns depending on the external bias, electrostatic environment, chip geometry, and magnetic field. Traditionally, such patterns were detected using nonlocal transport measurements [1-7], i.e., by measuring voltage drops between various leads other than the source and drain. Devised to study ballistic propagation of charge carriers in mesoscopic systems, these techniques were recently applied to investigate possible hydrodynamic behavior in ultrapure conductors [8-12], where the unusual behavior of the nonlocal resistance is often associated with viscosity of the electronic system [13-17].

Nonlocal resistance measurements have also been used to study edge states accompanying the quantum Hall effect [18-23]. While the exact nature of the edge states has been a subject of intense debate, the nonlocal resistance, $R_{N L}$, appears to be an intuitively clear consequence of the fact that the electric current flows along the sample edges and not through the bulk. Such a current would not be subject to exponential decay [24] exhibited by the bulk charge propagation leading to a much stronger nonlocal resistance.

In recent years the focus of the experimental work on electronic transport has been gradually shifting towards measurements at nearly room temperatures [6,8-10,21]. A particularly detailed analysis of the nonlocal resistance in a wide range of temperatures, carrier densities, and magnetic fields was performed on graphene samples [21]. Remarkably, the nonlocal resistance measured at charge neutrality remained strong well beyond the quantum Hall regime, with the peak value $R_{N L} \approx 1.5 \mathrm{k} \Omega$ at $B=12 \mathrm{~T}$ and $T=300 \mathrm{~K}$, three times higher than that at $T=10 \mathrm{~K}$.
In this Rapid Communication, we argue that the giant nonlocality observed in neutral graphene at high temperatures [21] can be directly attributed to the specific property of the graphene band structure: at the neutrality point, the two bands (the conductance and valence bands) touch creating a two-component-two-fluid-electronic system. This should be contrasted with the physics of doped graphene (where only a single band contributes to transport) as exemplified by the effect of magnetodrag [25,26], which is giant at the Dirac point and negligible in doped graphene. Similarly, the two-band physics leads to linear magnetoresistance [27,28] that is specific to neutral graphene. The two regimes (oneand two-fluid) are distinguished by temperature: the two-fluid regime occurs at high temperatures (relative to the chemical potential, $\mu \ll T$ ), while the single-fluid degenerate regime can be observed at low temperatures $(\mu \gg T)$.

The key physical feature of the two-fluid electronic system in intrinsic graphene is the existence of a neutral macroscopic current $\boldsymbol{j}_{I}[11,26,27]$ that may flow laterally to the electric current $\boldsymbol{J}$ once the external magnetic field is applied. Consider a standard Hall bar geometry with the spatially close source and drain leads (see Figs. 1-4). In the absence of the magnetic field the flow pattern of the electric current is the same in the two- and one-fluid systems: it is flowing mostly from source to drain with a weak diffusive spread into the rest of the sample (see Fig. 2 and Ref. [17]). The neutral current appearing in the two-fluid system is decoupled from the electric current and is practically undetectable. However, the effect of the magnetic field is dramatically different in the two cases. While the one-fluid system exhibits classical Hall effect (see Fig. 3), a compensated two-fluid system does not. Here the magnetic field couples the two macroscopic currents turning the neutral current to be lateral to $\boldsymbol{J}$. This way charge carriers may be transported to distant parts of the sample, where a nonlocal electric response is induced (again, by the magnetic field). The 


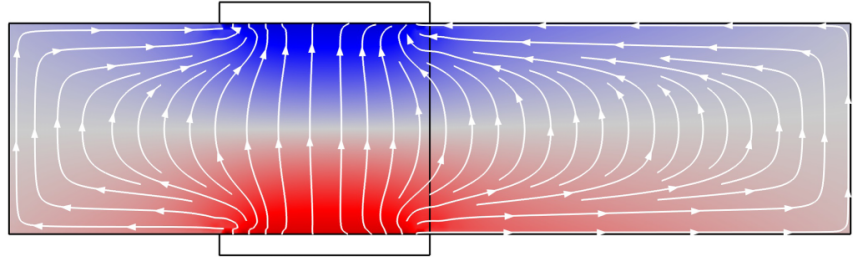

FIG. 1. Giant nonlocality in a compensated semimetal in a magnetic field $(B=2 \mathrm{~T})$. The arrows indicate the current flow and the color map shows the electrochemical potential (see the main text and Fig. 3 for specific parameters). The white rectangles are the source and drain leads. The nonlocal effect-the induced electrochemical potential difference between the top and bottom sides of the sample away from the source and drain and the associated edge currentappears due to the presence of the neutral quasiparticle flow that is coupled to the electric current by the magnetic field.

effect is strongly field dependent; in weak magnetic fields the resulting flow pattern is similar to the diffusive one albeit with the appreciable nonlocal resistance, while in strong fields a giant nonlocal pattern is formed where the current is flowing not only in the bulk, but also along the boundaries leading to strong nonlocal resistance (see Figs. 1, 4, and 5). Such patterns can be directly observed in experiments using the modern imaging techniques [29-31].

To highlight the difference between the one- and twocomponent systems, we briefly recall the macroscopic description of electronic transport in the standard (former) case. Allowing for nonuniform charge density, the linear relation between the electric current $\boldsymbol{J}$ and the external fields $\boldsymbol{E}, \boldsymbol{B}$ could be formulated as $[17,32,33]$

$$
r \boldsymbol{J}=\boldsymbol{E}+r_{H} \boldsymbol{e}_{\boldsymbol{B}} \times \boldsymbol{J}+(1 / e v) \nabla n,
$$

where $e>0$ is the unit charge, $v=\partial n / \partial \mu$ is the thermodynamic density of states (TDoS), $n$ is the carrier density, $\boldsymbol{e}_{\boldsymbol{B}}$ is the unit vector in the direction of the magnetic field, and $r$ and $r_{H}$ are the longitudinal and Hall resistivities. Within the Drude-like description, $r_{H}=\omega_{c} \tau r\left(\omega_{c}\right.$ is the cyclotron frequency and $\tau$ is the mean free path). The relation Eq. (1) is applicable to a wide range of electronic systems from simple metals $[34,35]$ to doped graphene [11,36]. The transport coefficients $r$ and $r_{H}$ could be treated as phenomenological or could be derived from the underlying kinetic theory $[11,32,37]$.

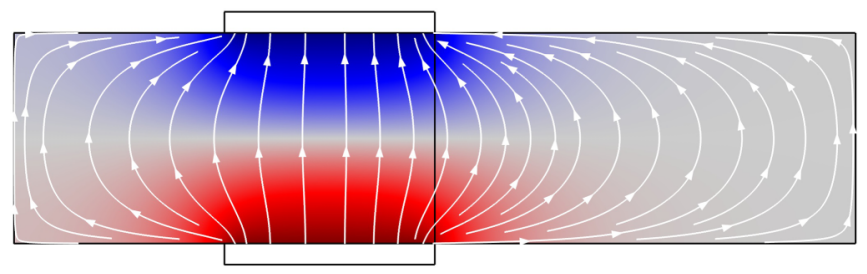

FIG. 2. Ohmic flow in two-component electronic systems at $B=0$. In the absence of the magnetic field the neutral quasiparticle current $\boldsymbol{j}_{I}$ is completely decoupled from the electric current $\boldsymbol{j}$. As a result, the latter exhibits the standard Ohmic flow profile similar to that of the one-component systems (see, e.g., Ref. [17]).

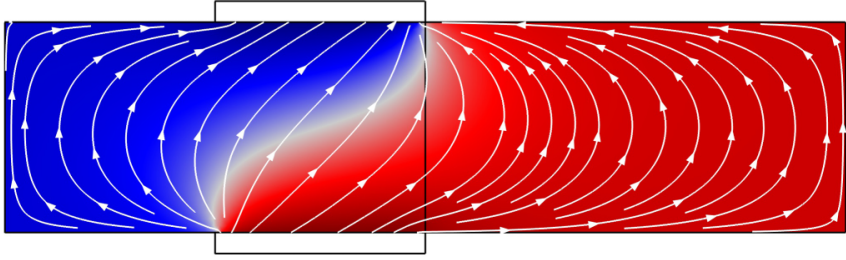

FIG. 3. Classical Hall effect in a one-component electronic system. The current density (shown by the arrows) and the electrochemical potential (shown by the color map) were obtained from Eqs. (1) for a sample of the width $W=1 \mu \mathrm{m}$ and length $L=4 \mu \mathrm{m}$ with the carrier density $n=10^{12} \mathrm{~cm}^{-2}$ at the temperature $T=240 \mathrm{~K}$ and in magnetic field $B=0.2 \mathrm{~T}$.

In addition to Eq. (1), the electric current satisfies the continuity equation, which for stationary currents reads

$$
\nabla \cdot J=0 .
$$

Charge density inhomogeneity induces electric field, so that Eq. (1) should be combined with the corresponding electrostatic problem. Most recent experiments were performed in gated structures, where the relation between the electric field and charge density simplifies [27,38]. In two-dimensional (2D) samples

$$
\boldsymbol{E}=\boldsymbol{E}_{0}-(e / C) \nabla n,
$$

where $C=\epsilon /(4 \pi d)$ is the gate-to-sample capacitance per unit area, $d$ is the distance to the gate, $\epsilon$ is the dielectric constant, and $\boldsymbol{E}_{0}$ is the external field.

In a two-terminal geometry, solving Eqs. (1) is a textbook problem. At $B=0$, the resulting electrochemical potential is governed by the relation of the mean free path to the system size, exhibiting either a flat (in short, ballistic samples) or linear (in long, diffusive samples) spatial profile. Most recently, these solutions were used as benchmarks in the imaging experiment [29] and the numerical solution of the hydrodynamic equations in doped graphene [17]. In an external magnetic field, the system exhibits the classical Hall effect, which in short samples is accompanied by nontrivial current flow patterns [39].

In a Hall bar geometry, the electric current still fills the whole sample, but decays exponentially [24] away from the direct path between source and drain. The resulting flow pattern was calculated (in the context of doped graphene) in Refs. [14,15,17]. In a magnetic field, the pattern gets skewed due to the classical Hall effect, but exhibits no qualitatively new features (see Fig. 3).

Let us now extend the transport equations (1) to a twocomponent system. Keeping in mind applications to graphene, we rewrite Eq. (1a) for the quasiparticles in the conduction band ("electrons") in the form

$$
-\boldsymbol{j}_{e}=e D v_{e} \boldsymbol{E}+\omega_{c} \tau \boldsymbol{j}_{e} \times \boldsymbol{e}_{\boldsymbol{B}}+D \nabla n_{e},
$$

where $j_{e}$ is the electron flow density (carrying the electric current $\boldsymbol{J}_{e}=-e \boldsymbol{j}_{e}$ ) and $v_{e}$ is TDoS. The "holes" (i.e., the quasiparticles in the valence band) are described by

$$
-\boldsymbol{j}_{h}=-e D v_{h} \boldsymbol{E}-\omega_{c} \tau \boldsymbol{j}_{h} \times \boldsymbol{e}_{\boldsymbol{B}}+D \nabla n_{h} .
$$




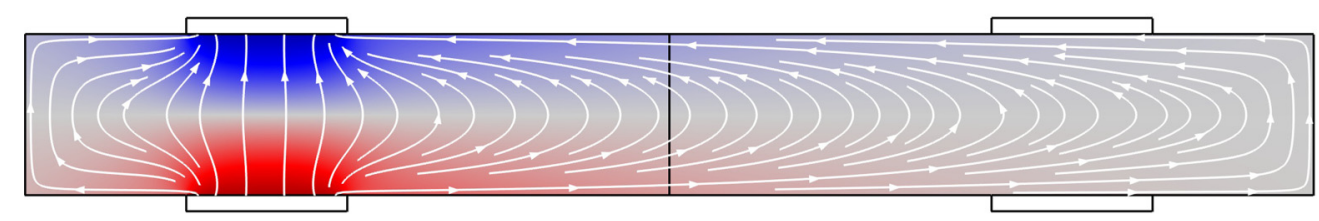

FIG. 4. Giant nonlocality in the Hall bar geometry. The white rectangles indicate external leads. The two leads on the left are the source and drain, while the two leads on the right are used to measure the nonlocal response, i.e., the induced electrochemical potential difference. The sample has a width $W=1 \mu \mathrm{m}$ and length $8 \mu \mathrm{m}$, with the distance between contacts $L=5 \mu \mathrm{m}$. The driving current is $I=0.1 \mu \mathrm{A}$. The flow pattern was computed for $B=0.8 \mathrm{~T}$ (cf. Fig. 1).

Here the electric current carried by the holes is $\boldsymbol{J}_{h}=e \boldsymbol{j}_{h}$ and TDoS may differ from that of electrons, $v_{h} \neq v_{e}$. For simplicity, we assume that the cyclotron frequency, mean free time, and diffusion constant for the two bands coincide (a generalization is straightforward, but does not lead to qualitatively new physics).
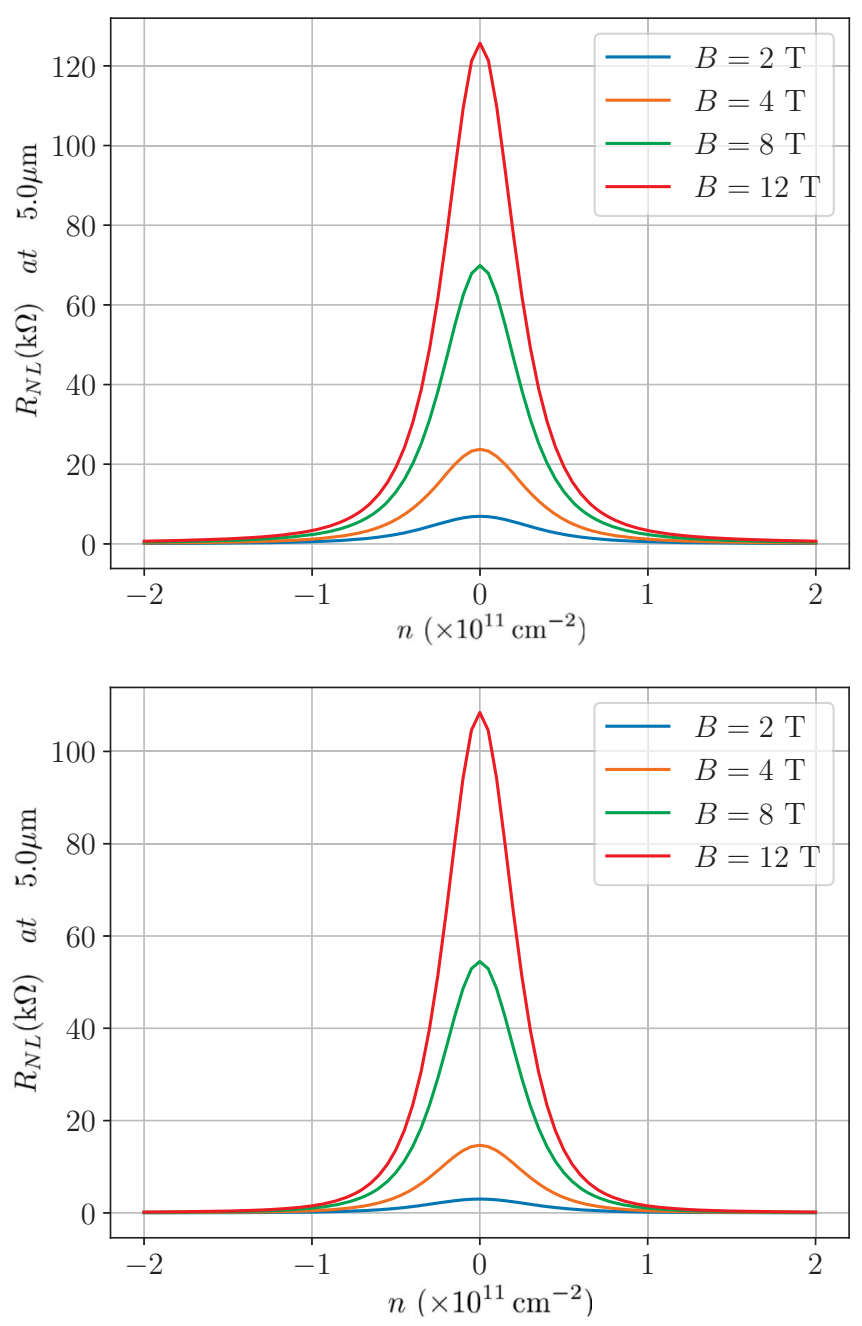

FIG. 5. Nonlocal resistance measured in the Hall bar geometry (see Fig. 4) as a function of carrier density. Top: Coulomb scatterers. Bottom: short-ranged impurities. The impurity model parameters are chosen to represent the mobility at $n=10^{11} \mathrm{~cm}^{-2}$ reported in Ref. [21]. The range of magnetic fields and carrier densities as well as the distance to the gate $(d=50 \mathrm{~nm})$ is taken from Ref. [21] (see Fig. 2).
The electric current in a two-component system is given by $\boldsymbol{J}=-e \boldsymbol{j}$, with $\boldsymbol{j}=\boldsymbol{j}_{e}-\boldsymbol{j}_{h}$. Introducing also the total quasiparticle flow $\boldsymbol{j}_{I}=\boldsymbol{j}_{e}+\boldsymbol{j}_{h}$, we find (cf. Ref. [37])

$$
\begin{aligned}
& \boldsymbol{j}+e D\left(v_{e}+v_{h}\right) \boldsymbol{E}+\omega_{c} \tau \boldsymbol{j}_{I} \times \boldsymbol{e}_{\boldsymbol{B}}+D \nabla n=0, \\
& \boldsymbol{j}_{I}+e D\left(v_{e}-v_{h}\right) \boldsymbol{E}+\omega_{c} \tau \boldsymbol{j} \times \boldsymbol{e}_{\boldsymbol{B}}+D \nabla \rho=0,
\end{aligned}
$$

where $n=n_{e}-n_{h}$ is the carrier density per unit charge (the charge density being $-e n$ ) and $\rho=n_{e}+n_{h}$ is the total quasiparticle density. The transport equations have to be supplemented by continuity equations reflecting the particle number conservation. The electric current satisfies Eq. (1b), but the total number of quasiparticles [40] can be affected by electron-hole recombination processes leading to a weak decay term in the continuity equation

$$
\nabla \cdot j_{I}=-\delta \rho / \tau_{R}
$$

where $\delta \rho$ is the deviation of the quasiparticle density from its equilibrium value and $\tau_{R}$ is the recombination time.

Under the assumption of electron-hole symmetry (e.g., at the charge neutrality point in graphene), $v_{e}=v_{h}$, we recover the phenomenological model of Ref. [27]. In the two-terminal geometry this model yields unsaturating linear magnetoresistance in classically strong fields [28].

Now we analyze the behavior of the phenomenological model (3) in the four-terminal Hall bar geometry. In the absence of the magnetic field, the system exhibits a typical Ohmic flow [14,15,17] (see the top panel in Fig. 2). Applying the field we find a qualitative change in the flow pattern-the emergence of a boundary flow and the associated electrochemical potential at the sample edges. Increasing the field leads to the nonlocal pattern growing until it fills the whole sample (see Figs. 1 and 4). Stronger fields essentially expel the current from the bulk with the charge flow being concentrated along the sample boundaries, which leads to strong nonlocal resistance.

The nonlocal flow pattern emerging in the magnetic field, Figs. 1 and 4, has to be contrasted with the vortices appearing in the viscous hydrodynamic flow (e.g., in doped graphene $[14,15,17,41])$. In the latter case, vorticity appears due to the constrained geometry of the flow and the particular boundary conditions [15,17,42]: neglecting Ohmic effects, the solution of the hydrodynamic equations can be obtained by introducing the stream function, which obeys a biharmonic equation independent of viscosity, which however affects the distribution of the electrochemical potential. In contrast, within the model (3) the "Ohmic" scattering represents the only source of dissipation and hence cannot be omitted. One can still introduce the stream function, but now it is determined 
not only by the sample geometry, but also by the Ohmic scattering and magnetic field. As a result, the flow pattern does not exhibit vortices, unlike those suggested recently for the hydrodynamic flow in intrinsic graphene [41] (in the absence of a magnetic field).

Having discussed the qualitative features of the charge flow in two-component systems, we now turn to a quantitative calculation of nonlocal resistance in graphene. Although the model (3) is applicable to any semimetal, graphene is a by far better studied material with readily available experimental values for model parameters. Here we use the data measured in Refs. $[8,9,21,26,43]$ and theoretical calculations of Refs. [11,12,26,37,41].

TDoS of the quasiparticles in graphene has been evaluated in, e.g., Refs. [11,12,36,37], and has the form

$$
v_{e}+v_{h}=2 \mathcal{T} /\left(\pi v_{g}^{2}\right), \quad v_{e}-v_{h}=2 \mu /\left(\pi v_{g}^{2}\right),
$$

where $\mu$ is the chemical potential, $v_{g}$ is the quasiparticle velocity in graphene, and $\mathcal{T}=2 T \ln [2 \cosh (\mu / 2 T)]$. The generalized cyclotron frequency is $\omega_{c}=e B v_{g}^{2} /(c \mathcal{T})$ and the diffusion coefficient has the usual form $D=v_{g}^{2} \tau / 2$. At charge neutrality, $\mu=0$ and $\mathcal{T}=2 T \ln 2$, while in the degenerate regime $\mathcal{T}(\mu \gg T)=\mu$. The latter confirms that all coefficients in Eqs. (3a) and (3b) become identical with doping. Similarly, the continuity equations (1b) and (3c) should coincide in the degenerate regime. In graphene this happens by means of the fast decay of the recombination rate [26]. Close to neutrality we assume

$$
\tau_{R}^{-1}=g^{2} T / \cosh (\mu / T),
$$

where $g$ is determined by the corresponding matrix element. The above expression [26] reflects the exponential decay of the two-band physics away from charge neutrality, which is responsible for the fast decay of $R_{N L}$ as a function of carrier density [21] (see Fig. 5). Finally, the mean-free time, $\tau$, in graphene is a nontrivial function of temperature and carrier density $[11,12,36,43,44]$, which strongly depends on the model of the impurity potential [45-50]. However, these dependencies are typically not exponential and hence do not affect the exponential decay of the nonlocal resistance.

In Fig. 5 we demonstrate the decay of $R_{N L}$ for two impurity models - the Coulomb scatterers and short-ranged impurities-showing nearly identical behavior. Such robustness of the model (3) with respect to the functional dependence of the mean-free time justifies the inaccuracy of our description of electronic transport in graphene, where close to charge neutrality the resistivity is strongly affected by electron-electron interaction. The data shown in Fig. 5 were obtained by solving Eqs. (3) in the Hall bar geometry of Fig. 4 using the estimate [41] for the recombination length scale, $\ell_{R}=v_{g} \tau_{R} \approx 10 \mu \mathrm{m}$ (a previous calculation of Ref. [26] put it at a smaller value $1.2 \mu \mathrm{m}$ ), which leads to similar results for the nonlocal resistance, but with a smaller peak value at charge neutrality.

To summarize, we have considered a two-fluid model of a nearly compensated semimetal in an idealized Hall bar geometry. We have shown that this system exhibits a strong nonlocal response when subjected to an external magnetic field (see Fig. 5). The effect is specific to the neutrality point and shows a rapid decay with doping [as a function of either the charge density (see Fig. 5), or the chemical potential]. The physical origin of the effect is the existence of the neutral quasiparticle flow that is coupled to the electric current by the magnetic field [26,27] and may carry the charge along the Hall bar (see Fig. 4), leading to the nonlocal effectthe electrochemical potential difference (voltage) between the pair of contacts that are spatially distant from the source and drain.

Our results provide a qualitative explanation for the giant nonlocality reported in Ref. [21] in graphene near charge neutrality, where the touching conductance and valence bands form a two-fluid electronic system. Having considered the idealized geometry (and importantly, boundary conditions), we have captured the main features of the effect: strong nonlocal response appears at charge neutrality only when the system is subjected to the magnetic field and its rapid decay away from the Dirac point. Our theory does not involve spinrelated phenomena including the effect of Zeeman splitting invoked in Ref. [21]. The latter should be independent of the field direction; however, the effect was not observed in the nearly parallel field studied in Ref. [51]. Assuming the $g$ factor to be equal to 2 , we estimate the Zeeman splitting as $E_{z} \approx 0.35 \mathrm{meV} \approx 4 \mathrm{~K}$ at $B=10 \mathrm{~T}$. The corresponding residual quasiparticle density (at $T=0$ ) is given by $\rho_{Q}=E_{z}^{2} /\left(4 \pi v_{g}^{2}\right) \approx 2.2 \times 10^{6} \mathrm{~cm}^{-2}$. As a result, at temperatures and carrier densities typical to nonlocal measurements discussed here we expect the effects of Zeeman splitting to be much smaller not only than our results, but also the data of Ref. [21].

Our calculations in the idealized rectangular sample yield $R_{N L}$ that is orders of magnitude stronger than the theoretical explanation of Ref. [21]. At the same time, our $R_{N L}$ exceeds the experimental data of Ref. [21] as well. In realistic systems, we expect $R_{N L}$ to be suppressed from the values obtained in our idealized geometry with ideal boundary conditions for the following reasons. Firstly, by ignoring the effects of electron-electron interaction, we strongly underestimate the usual resistivity of intrinsic graphene. Secondly, we ignore viscous effects. Thirdly, carrier population in real graphene does not vanish "at neutrality" due to electrostatic potential fluctuations [51] with the minimal concentration often as high as $10^{10} \mathrm{~cm}^{-2}$, essentially cutting off the lower density range around the peak in Fig. 5. Finally, Eq. (5) is a rather crude estimate that needs to be improved. Such effects are expected to reduce $R_{N L}$ down to the values of the same order of magnitude as the experimental data of Ref. [21]. For graphene, a more precise calculation involving solution of the full system of hydrodynamic equations near charge neutrality is required to reach perfect agreement with the data. The present approach shows that the effect is more general and does not rely on the additional assumptions of electronic hydrodynamics.

The authors are grateful to I. V. Gornyi, A. D. Mirlin, J. Schmalian, J. A. Sulpizio, M. Schütt, A. Shnirman, and Y. Tserkovnyak for fruitful discussions. This work was supported by the German Research Foundation DFG within FLAG-ERA Joint Transnational Call (Project GRANSPORT), by the European Commission under the EU Horizon 2020 MSCA-RISE-2019 program (Project No. 873028 
HYDROTRONICS), and by the Russian Science Foundation (Project No. 17-12-0) (M.T.). B.N.N. acknowledges the support by the MEPhI Academic Excellence Project, Contract No. 02.a03.21.0005.
[1] W. J. Skocpol, P. M. Mankiewich, R. E. Howard, L. D. Jackel, D. M. Tennant, and A. D. Stone, Phys. Rev. Lett. 58, 2347 (1987).

[2] H. van Houten, C. W. J. Beenakker, J. G. Williamson, M. E. I. Broekaart, P. H. M. van Loosdrecht, B. J. van Wees, J. E. Mooij, C. T. Foxon, and J. J. Harris, Phys. Rev. B 39, 8556 (1989).

[3] A. K. Geim, P. C. Main, P. H. Beton, P. Streda, L. Eaves, C. D. W. Wilkinson, and S. P. Beaumont, Phys. Rev. Lett. 67, 3014 (1991).

[4] K. L. Shepard, M. L. Roukes, and B. P. Van der Gaag, Phys. Rev. Lett. 68, 2660 (1992).

[5] Y. Hirayama, A. D. Wieck, T. Bever, K. von Klitzing, and K. Ploog, Phys. Rev. B 46, 4035 (1992).

[6] G. Mihajlović, J. E. Pearson, M. A. Garcia, S. D. Bader, and A. Hoffmann, Phys. Rev. Lett. 103, 166601 (2009).

[7] R. V. Gorbachev, J. C. W. Song, G. L. Yu, A. V. Kretinin, F. Withers, Y. Cao, A. Mishchenko, I. V. Grigorieva, K. S. Novoselov, L. S. Levitov et al., Science 346, 448 (2014).

[8] D. A. Bandurin, I. Torre, R. Krishna Kumar, M. Ben Shalom, A. Tomadin, A. Principi, G. H. Auton, E. Khestanova, K. S. Novoselov, I. V. Grigorieva et al., Science 351, 1055 (2016).

[9] D. A. Bandurin, A. V. Shytov, L. S. Levitov, R. K. Kumar, A. I. Berdyugin, M. Ben Shalom, I. V. Grigorieva, A. K. Geim, and G. Falkovich, Nat. Commun. 9, 4533 (2018).

[10] A. I. Berdyugin, S. G. Xu, F. M. D. Pellegrino, R. K. Kumar, A. Principi, I. Torre, M. B. Shalom, T. Taniguchi, K. Watanabe, I. V. Grigorieva, M. Polini, A. K. Geim, and D. A. Bandurin, Science 364, 162 (2019).

[11] B. N. Narozhny, I. V. Gornyi, A. D. Mirlin, and J. Schmalian, Ann. Phys. 529, 1700043 (2017).

[12] A. Lucas and K. C. Fong, J. Phys: Condens. Matter 30, 053001 (2018).

[13] I. Torre, A. Tomadin, A. K. Geim, and M. Polini, Phys. Rev. B 92, 165433 (2015).

[14] L. Levitov and G. Falkovich, Nat. Phys. 12, 672 (2016).

[15] G. Falkovich and L. Levitov, Phys. Rev. Lett. 119, 066601 (2017).

[16] F. M. D. Pellegrino, I. Torre, and M. Polini, Phys. Rev. B 96, 195401 (2017).

[17] S. Danz and B. N. Narozhny, 2D Mater. 7, 035001 (2020).

[18] P. L. McEuen, A. Szafer, C. A. Richter, B. W. Alphenaar, J. K. Jain, A. D. Stone, R. G. Wheeler, and R. N. Sacks, Phys. Rev. Lett. 64, 2062 (1990).

[19] J. K. Wang and V. J. Goldman, Phys. Rev. B 45, 13479 (1992).

[20] A. Roth, C. Brune, H. Buhmann, L. W. Molenkamp, J. Maciejko, X.-L. Qi, and S.-C. Zhang, Science 325, 294 (2009).

[21] D. A. Abanin, S. V. Morozov, L. A. Ponomarenko, R. V. Gorbachev, A. S. Mayorov, M. I. Katsnelson, K. Watanabe, T. Taniguchi, K. S. Novoselov, L. S. Levitov et al., Science 332, 328 (2011).

[22] X.-P. Zhang, C. Huang, and M. A. Cazalilla, 2D Mater. 4, 024007 (2017).
[23] K. Komatsu, Y. Morita, E. Watanabe, D. Tsuya, K. Watanabe, T. Taniguchi, and S. Moriyama, Sci. Adv. 4, eaaq0194 (2018).

[24] L. J. van der Pauw, Philips Tech. Rev. 20, 223 (1958).

[25] R. V. Gorbachev, A. K. Geim, M. I. Katsnelson, K. S. Novoselov, T. Tudorovskiy, I. V. Grigorieva, A. H. MacDonald, S. V. Morozov, K. Watanabe, T. Taniguchi et al., Nat. Phys. 8, 896 (2012).

[26] M. Titov, R. V. Gorbachev, B. N. Narozhny, T. Tudorovskiy, M. Schütt, P. M. Ostrovsky, I. V. Gornyi, A. D. Mirlin, M. I. Katsnelson, K. S. Novoselov et al., Phys. Rev. Lett. 111, 166601 (2013).

[27] P. S. Alekseev, A. P. Dmitriev, I. V. Gornyi, V. Y. Kachorovskii, B. N. Narozhny, M. Schütt, and M. Titov, Phys. Rev. Lett. 114, 156601 (2015).

[28] G. Y. Vasileva, D. Smirnov, Y. L. Ivanov, Y. B. Vasilyev, P. S. Alekseev, A. P. Dmitriev, I. V. Gornyi, V. Y. Kachorovskii, M. Titov, B. N. Narozhny et al., Phys. Rev. B 93, 195430 (2016).

[29] L. Ella, A. Rozen, J. Birkbeck, M. Ben-Shalom, D. Perello, J. Zultak, T. Taniguchi, K. Watanabe, A. K. Geim, S. Ilani et al., Nat. Nanotechnol. 14, 480 (2019).

[30] M. J. H. Ku, T. X. Zhou, Q. Li, Y. J. Shin, J. K. Shi, C. Burch, L. E. Anderson, A. T. Pierce, Y. Xie, A. Hamo, U. Vool, H. Zhang, F. Casola, T. Taniguchi, K. Watanabe, M. M. Fogler, P. Kim, A. Yacoby, and R. L. Walsworth, Nature 583, 537 (2020).

[31] J. A. Sulpizio, L. Ella, A. Rozen, J. Birkbeck, D. J. Perello, D. Dutta, M. Ben-Shalom, T. Taniguchi, K. Watanabe, T. Holder et al., Nature (London) 576, 75 (2019).

[32] E. M. Lifshitz and L. P. Pitaevskii, Physical Kinetics (Pergamon, London, 1981).

[33] B. N. Narozhny, I. L. Aleiner, and A. Stern, Phys. Rev. Lett. 86, 3610 (2001).

[34] J. M. Ziman, Principles of the Theory of Solids (Cambridge University Press, Cambridge, 1965).

[35] G. Giuliani and G. Vignale, Quantum Theory of the Electron Liquid (Cambridge University Press, Cambridge, UK, 2005).

[36] M. I. Katsnelson, Graphene (Cambridge University Press, Cambridge, UK, 2012).

[37] B. N. Narozhny, Ann. Phys. 411, 167979 (2019).

[38] I. L. Aleiner and B. I. Shklovskii, Phys. Rev. B 49, 13721 (1994).

[39] A. Shik, J. Phys.: Condens. Matter 5, 8963 (1993).

[40] M. S. Foster and I. L. Aleiner, Phys. Rev. B 79, 085415 (2009).

[41] H.-Y. Xie and A. Levchenko, Phys. Rev. B 99, 045434 (2019).

[42] E. I. Kiselev and J. Schmalian, Phys. Rev. B 99, 035430 (2019).

[43] P. Gallagher, C.-S. Yang, T. Lyu, F. Tian, R. Kou, H. Zhang, K. Watanabe, T. Taniguchi, and F. Wang, Science 364, 158 (2019).

[44] B. N. Narozhny, M. Titov, I. V. Gornyi, and P. M. Ostrovsky, Phys. Rev. B 85, 195421 (2012).

[45] T. Ando, J. Phys. Soc. Jpn. 75, 074716 (2006).

[46] N. Shon and T. Ando, J. Phys. Soc. Jpn. 67, 2421 (1998). 
[47] K. Nomura and A. H. MacDonald, Phys. Rev. Lett. 96, 256602 (2006).

[48] V. V. Cheianov and V. I. Fal'ko, Phys. Rev. Lett. 97, 226801 (2006).

[49] I. L. Aleiner and K. B. Efetov, Phys. Rev. Lett. 97, 236801 (2006).
[50] P. M. Ostrovsky, I. V. Gornyi, and A. D. Mirlin, Phys. Rev. B 74, 235443 (2006).

[51] F. Chiappini, S. Wiedmann, M. Titov, A. K. Geim, R. V. Gorbachev, E. Khestanova, A. Mishchenko, K. S. Novoselov, J. C. Maan, and U. Zeitler, Phys. Rev. B 94, 085302 (2016). 